\title{
Reconfigurable System for Wireless Power Transfer (WPT) and Near Field Communications (NFC)
}

\author{
Josep. I. Cairó, Jordi Bonache, Member, IEEE, Ferran Paredes, Member, IEEE, and Ferran Martín, \\ Fellow, IEEE
}

\begin{abstract}
In this work, it is investigated the possibility of using near field communication (NFC), normally applied for communicating two proximity devices, to power-up external circuitry, such as low power wireless sensors, or energy scavenging devices. For this purpose, the possibility of combining both features in a single system, able to communicate with neighboring devices via NFC and to transfer power from one device to another (wireless power transfer -WPT), has been studied. After taking considerations on antenna design, both for NFC and WPT, a reconfigurable circuitry optimized for data and energy transfer is proposed. The circuit provides optimal close proximity wireless magnetic communications, and is able to use the same device for optimal energy transfer. This feature has been implemented and demonstrated using PCB antennas, NFC reader with a mobile phone or NFC-tag and a circuitry for charging an external Io-Li battery. The results show an improvement of the energy transfer when such circuit capability is turned on.
\end{abstract}

Index Terms-Coupling, wireless power transfer (WPT), nearfield communications (NFC), switching.

\section{INTRODUCTION}

$\mathrm{N}$ ear field communications (NFC) are being used for identification or payment transactions, in ePassport or travel cards, and also in applications such as embedded sensors [1]. In these latter applications, NFC tags can be totally passive, such as the implantable glucose sensors in [2], but other NFC tags require small batteries, such as those described in [3], where NFC enabled devices collect patient data. In those cases, a small battery might be required, so one optimum case would be to do the charging from the same NFC reader. One can say that NFC equipped with WPT techniques can be seen as extra capability for applications such NFC sensors devices. A battery used as auxiliary power supply, provides extra capability for applications in such sensor networks. Reference [4] provides an example of the use of a $1 \mathrm{~KB}$ MIFARE Classic Tag IC compliant with NFC ISO/IEC 14443 Type A standard, where functionality of data logger justifies the use of a rechargeable battery.

In terms of communications, NFC is a technology for applications which demand relatively low data rate (106 kbps).
NFC can achieve data rates up to $424 \mathrm{kbps}$ at $22 \mathrm{~cm}$ (according to the standard) or $848 \mathrm{kbps}$ at closer proximity, so less coupling time is required for the same transaction. Even so, since NFC is based on inductive wireless data transfer, it can also be an attractive solution for energy transfer between separated devices. However, some limitation arises when observing the purpose for which NFC was conceived, that is, information (or data) data transfer. So, in this case, the circuit designed for this purpose (data transfer) is not being optimized for efficiently transferring power between devices.

Some previous works [5] have already studied the coexistence between NFC and WPT, where the interference between the NFC transmitter coil and the WPT coils is analyzed. Although involving different frequencies, NFC frequency $(13.56 \mathrm{MHz})$ coincides with the second harmonic of WPT (6.78 MHz). Hence, compatibility issues may arise. In [6], the coexistence of both wireless systems is analyzed in terms of induced voltage by the NFC tag, in the presence of WPT system.

In near-field, the mutual inductance at high frequency of both antenna coils acts as a loosely (roughly) magnetically coupled transformer, where energy is magnetically induced and propagated from the primary to the secondary coil. Therefore, unlike far-field antennas, which are characterized by gain, directivity, and radiation pattern, the coil antennas in near-field are best characterized by the coupling coefficient, $k$, between the reader and the tag coils, by the $H$-field at the receiving tag coil, by the inductance, $L$, of the coils, and by the unloaded quality factor, $Q$, of the antenna.

An inductive link consists of two weakly coupled resonant circuits, where coil coupling conditions can be very weak due to possible misalignment, large coil separation, or a very small pick-up coil diameter. A way to transfer power in near-field NFC is to add a parallel capacitor to create a resonance. In the case of very short distances, high power transfer efficiency can be achieved (on the order of $90 \%$ for very short lengths, i.e., 1$3 \mathrm{~cm}$ ) [7]. However, the efficiency of such techniques drops drastically for higher distances, decaying at a ratio of $1 / r^{6}, r$ being the link distance.

The authors are with CIMITEC, Departament d'Enginyeria Electrònica, Universitat Autònoma de Barcelona, 08193 Bellaterra, Spain. E-mail: josepignasi.cairo@e-campus.uab.cat.

(C) 2017 IEEE. Personal use of this material is permitted. Permission from IEEE must be obtained for all other uses, in any current or future media, including reprinting/republishing this material for advertising or promotional purposes, creating new collective works, for resale or redistribution to servers or lists, or reuse of any copyrighted component of this work in other works. 
The efficiency of the voltage transfer ratio between two coil antennas can be increased significantly with high $Q$ circuits. The antenna coil design in such systems is mainly influenced by low cost, low profile, and electrically small size restrictions. In relation to the bandwidth (BW) requirement for tag coils, it is not critical in most NFC applications. However, that is not true when coming to apply the antenna for NFC communications, where data is transferred at higher data rates.

In practice, the inductive link can be implemented by one of the four possible combinations of series or parallel tuned topology between primary and secondary coil antennas. In [8], the coupling between inductances to realize maximum WPT, introducing their conjugate image impedances, has been studied. Optimal antenna coil size design, where the internal area of the coil can be exploited to enhance the $\mathrm{H}$-field, has been introduced in [9], using an unequal spacing between turns of the coil, that allows to adjust the self-inductance $(L)$ without detuning its resonance frequency $\left(f_{r}\right)$. Other studies have introduced optimization methods with tunable metamaterials for performance improvement in WPT, but some extra devices are required in system integration [10].

For data communication applications, to obtain better reading range, it is recommended to maximize the induced voltage $\left(V_{\text {ind }}\right)$ in the receiver tag, responsible for activating the tag chip. To maximize energy transfer, it is desired to maximize the power efficiency from primary to secondary circuit. Several possibilities to optimize such parameters are analyzed:

- A first approach is to improve the coupling factor $(k)$ maintaining $L$ [11]. It implies improved coverage and antenna size reduction due to increase in induced voltage $V_{\text {ind }}$ as a function of current $I$ in the reader:

$$
V_{\text {ind }}=j \omega M_{12} I=j \omega k \sqrt{L_{\text {tag }} L_{r d}} I
$$

$M_{12}$ being the mutual inductance between the primary coil ( $L_{R r d}$ reader) and the secondary coil ( $L_{t a g}$ Tag). As shown in [11], an optimum $k$ design, while keeping $L$ constant, results in $25 \%$ reduction in $Q$-factor, which implies an increase in antenna loss and reduced efficiency.

- A second approach is to improve the propagating $H$-field between reader and tags; so following Faraday's law $V_{\text {ind }}=$ $-\frac{d \Phi(t)}{d t}, \Phi$ being the magnetic flux flowing through the area of the Tag $\left(A_{T a g}\right)$, the induced voltage is increased as:

$$
V_{\text {ind }}(\omega)=\emptyset j \omega=B A_{\text {Tag }} j \omega=j \omega \mu_{0} N_{\text {Tag }} A_{\text {Tag }} H
$$

$B$ being the induced magnetic flux, and $N_{T a g}$ the number of turns of the tag coil. However, to improve $H$ (increase number of loops) in the coil, it will lower $Q$ of the reader coil (this assumes a constant $L$, which cannot change since the chip has a fixed capacitance, and at resonance we need to maintain inductance, since $\left.Q=\omega L / /_{R} \quad[10,11]\right)$. Additionally there is a limitation in maximum $H$-field one can reach. So for NFC, the standard [12] defines for Europe maximum field strength of $60 \mathrm{~dB} \mu \mathrm{A} / \mathrm{m}$ at $13.56 \mathrm{MHz}$ for a transmitter at a distance of $10 \mathrm{~m}$, and the bandwidth is \pm 900
$\mathrm{kHz}$, which will limit the maximum $H$-field generated by the loop antenna.

- A third approach is to design smaller antennas. If the loop area is reduced by half, this could be compensated by an increase in the number of turns by 2 to achieve the same induced voltage (eq.2).

There are, therefore, several possibilities to improve data transfer rate (needed in NFC) and energy transfer (needed for WPT) between reader and receiver, but none of them provides an optimum solution for both at the same time. So, a trade-off, in order to avoid degrading one parameter by improving the other one, is needed. Therefore, the possibility of improving, both the data and energy transfer between two coils (reader and receiver or tag) simultaneously is studied, and a solution is proposed in this paper.

\section{DUAL APPROACH BETWEEN POWER AND COMMUNICATIONS TRANSFER}

In this section, the possible improvements to be applied in a HF coupled circuit, when being utilized as communication system and as energy transfer circuit, are studied. The focus is on analyzing the design of the matching circuit between driver and loop antenna, and introducing the key points of the HF antenna design, which also affect the matching network. A practical set up to test some possible solutions is proposed and developed.

\section{A. Matching network considerations}

The matching network, normally composed by two or three capacitors, is responsible for matching the output impedance of the IC driver $\left(R_{P A}\right)$ to the antenna impedance, in order to transfer maximum power from the NFC-IC to the antenna (see Fig. 1). Since the driver is normally a class D-E high efficiency power amplifier, its output impedance may vary from $2 \Omega$ to $5 \Omega$ (matching network transformation is introduced in Fig.2).

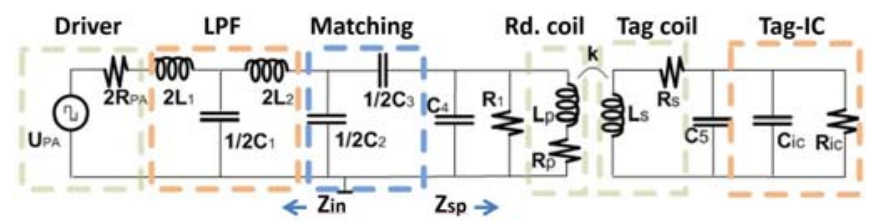

Fig. 1. Equivalent single ended circuit for the matching network of the NFC transmitter, when linked with receiver tag antenna coil $\left(C_{2}=1100 \mathrm{pF}\right.$, $\left.C_{3}=330 \mathrm{pF}\right)$.

Since antennas have inductive performance, a simplified Ltopology matching network with two capacitors (see Fig. 1) can be used. This is advantageous for power efficiency since a capacitor can be fabricated nearly lossless for HF (type COG or NPO). The NFC antenna has its first and fundamental parallel self-resonance above the carrier frequency, so we can assume it is a complex inductive load. A combination of LC in T-shape is added between driver and matching, that smoothes the fast switching driver output to perform a filtering functionality, as shown in Fig.1. 


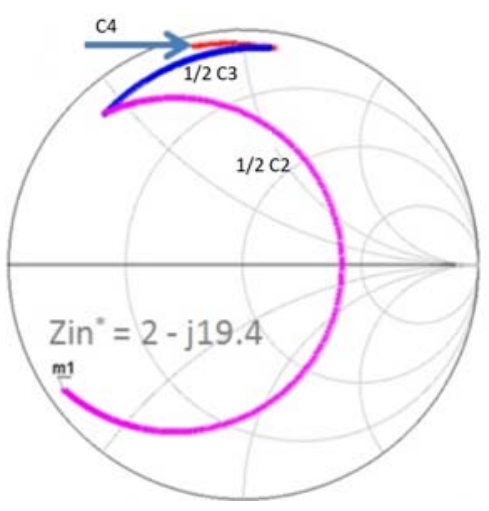

Fig. 2. Impedance transformation from ID driver to the matching circuit, including the effects of each matching capacitor in the Smith Chart diagram.

The IC impedance can only be matched at a single operating frequency, or within a small frequency band, depending on the quality factor $(Q)$. The PA will then feed just a small amount of effective power $P_{E}$ that will compensate damping losses. For a high H-field, it is desired to increase antenna current. The major contribution of the antenna current is the reactive power of the oscillating energy $W_{M}$, for which the quality factor is responsible $\left(Q=\frac{W_{M}}{P_{E}} \omega=\frac{I^{2} L_{S}}{I^{2} R_{S}} \omega=\frac{L_{S} \omega}{R_{S}}\right), W_{M}$ being the magnetizing energy and $R_{S}$ the series resistance coil.

For a given injected power from driver PA stage into the antenna, a high $H$-field strength can be achieved (higher reactive power), and also low losses $R_{s}$, as desired (optimum situation for power transfer between coils) with high-Q circuit. On the other hand, it is well known that for optimum data rate between two inductively coupled circuits, a low Q factor is required, since bandwidth needs to be maximized.

\section{B. Dual solution for the matching network}

A method for HF antenna design, taking into consideration the two requirements presented in this work is discussed. There are different premises to consider when defining the matching network for the tag and reader antenna. For tag antenna design, the impedance presented at antenna terminals will depend on the equivalent chip resistance $\left(R_{I C}\right)$ and chip capacitance $\left(C_{I C}\right)$, which depend on technology and number of transistor gates, and also on the assembly chip package (bonding wires, flipchip). In this case, the tag antenna is optimized to match the IC. For the reader, there are two situations that will influence the design of the matching network: as a wireless communication system, such as NFC, or as a charging system, such as WPT. In each case, for practical calculations, it is desirable to simplify the impedance seen by the antenna.

To test the antenna performance in the dual approach, two $\mathrm{HF}$ antennas have been designed. Electromagnetic simulations are conducted using the method of moments implemented in the Keysight ADS Momentum simulation tool, to complement the calculations and elaborate the antenna design. The comparison between analytical expressions and Momentum simulations are shown in table 1.
TABLE I

COIL DESIGN COMPARING ANALYSIS AND SIMULATION

\begin{tabular}{|c|c|c|c|c|c|}
\hline \multirow{2}{*}{ Sample } & \multirow{2}{*}{$\begin{array}{l}\text { Outline } \\
(\mathrm{mm})\end{array}$} & \multicolumn{2}{|c|}{ ADS } & \multicolumn{2}{|c|}{ Analytic } \\
\hline & & $\mathrm{L}(\mathrm{nH})$ & Q & $\mathrm{L}(\mathrm{nH})$ & Q \\
\hline$\overline{L_{A}}$ & $30 \times 30$ & 586 & 86 & 578 & 117 \\
\hline$L_{B}$ & $93 \times 60$ & 1080 & 81 & 994 & 118 \\
\hline
\end{tabular}

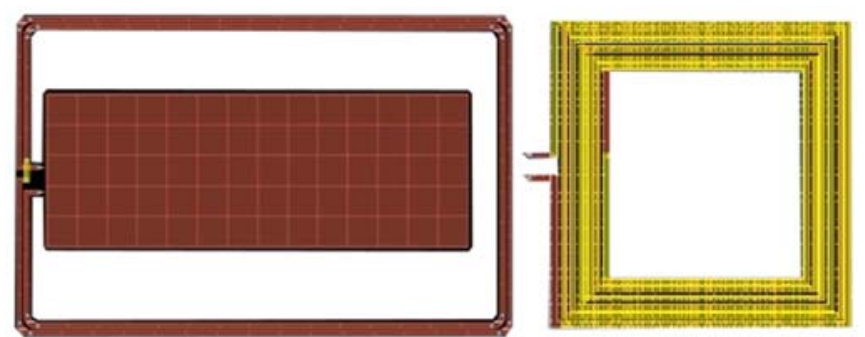

Fig. 3. Layout of two different implemented coils, $\mathrm{L}_{\mathrm{B}}, \mathrm{N}=2$ one layer (left), and $\mathrm{L}_{\mathrm{A}}$ with two layers $\mathrm{N}=6+6$ (right).

Two antennas have been designed (see Fig.3), adopting $L_{B}$ $(1080 \mathrm{nH})$ for the reader loop antenna, which already includes a mobile ground plane to locate the reader circuitry (see Fig.3), and $L_{A}$ for the receiver. Coil antennas are built on FR4 material with thickness $t=0.8 \mathrm{~mm}$ and $1.6 \mathrm{~mm}$, relative permittivity $\varepsilon_{r}$ $=3.55$ and $\varepsilon_{r}=6$, track gap $g=0.3 \mathrm{~mm}$, track width $w=0.7$ $\mathrm{mm}$, and number of turns $N=6$ and 2. Metal conductivity is $\sigma=5.8 \cdot 10^{7}[\mathrm{~S} / \mathrm{m}]$, and its thickness is $t_{C u}=18 \mu \mathrm{m} . L_{B}$ has an equivalent parallel $\mathrm{AC}$ resistance of

$$
R_{e q}=L_{B} \omega Q=7,4 k \Omega
$$

The DC series loop series resistance $R_{D C}(470 \mathrm{~m} \Omega$, as inferred by simulation) is converted into equivalent parallel resistance $\left(R_{p}\right)$ using equation (4). In our case, it can be approximated by $R_{p}=\frac{X_{L 1}{ }^{2}}{R_{D C}},\left(R_{p}=18 \mathrm{k} \Omega\right.$, see Fig.4). The loop AC resistance that would be measured at the working frequency $R_{A C}$ will be the one given in (5).

$$
\begin{gathered}
R_{p}=\frac{X_{L 1}^{2}+R_{D C} X_{L 1}}{R_{D C}} \\
R_{e q}=\frac{R_{p} R_{A C}}{R_{p}+R_{A C}} ; \quad R_{A C}=\frac{R_{p} R_{e q}}{R_{p}-R_{e q}}=12,5 \mathrm{k} \Omega
\end{gathered}
$$

Such resistance would be the equivalent at the working frequency.

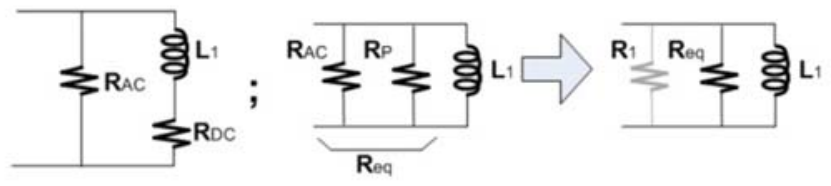

Fig. 4. Equivalent circuit of the loop antenna

For NFC, with a maximum data rate of $\mathrm{R}=848 \mathrm{Kbps}$, the required quality factor from the antenna should be $\mathrm{Q}=f_{\mathrm{r}} / B_{\omega}=16$. It is necessary then to reduce the antenna $\mathrm{Q}$ value from 86 to a value close to the previous one, e.g., 20, in order to comply with data communications requirements. This 
can be done by incorporating a parallel resistance with the coil $\left(R_{l}\right.$, see Fig.4). In order to do so, the driver circuit should see at the antenna terminals a parallel resistance of value $\mathrm{R}_{\mathrm{T}}=\mathrm{Q} \cdot \mathrm{X}_{\mathrm{LA}}=1 \mathrm{k} \Omega$. Hence, the resistance to place in parallel with the coil will be $R_{1}=1.2 \mathrm{k} \Omega$.

In the case of WPT, a high Q would be preferred, so keeping the high $\mathrm{Q}$ value, in this case 81 , is convenient. No additional resistance needs to be added.

To summarize, it is possible to achieve optimum matching for NFC or WPT by considering the possibility of modifying the quality factor of antenna by means of a switchable resistance.

\section{RECONFIGURABLE DESIGN FOR THE READER ANTENNA}

So far, it has been shown that different antenna matching conditions to comply with different requirements are needed. From this point, a solution coping with the two considered necessities is presented, and some formulation is provided.

\section{A. Presented trade-off}

The tradeoff can be summarized as follows: (i) for proximity wireless communications, low coupling coefficient $\left(k_{\text {low }}\right)$ and low-quality factor $\left(Q_{\text {low }}\right)$ are nedded; (ii) conversely, for WPT, higher coupling coefficient $\left(k_{\text {high }}\right)$ and higher quality factor $\left(Q_{h i g h}\right)$ are a due. Consequently, there is not an optimum solution compatible with both requirements, once a pair of coupling antenna coils is designed.

In NFC the coupling factor between the two antennas is small, because the system is designed to reach maximum distance. Tag responses can reach up to $60 \mathrm{~dB}$ below the voltage generated by the reader. In a WPT system, closer proximity between the antennas is considered, resulting in higher power coupling. However, the coils are usually loosely coupled due to the absence of a common magnetic core to confine and guide most of the magnetic flux, such as occurs in a transformer.

\section{B. Solution proposed for bandwidth and efficiency tuning}

A solution able to accommodate both presented requirements with the minimum design effort is presented in this subsection. The focus is the matching network implementation. The matching in Fig. 1 already includes a resistor that reduces the quality factor of the reader inductor, accomplishing the conditions of NFC wideband communications. The idea proposed here consists of using the same reader for wideband communications and for power transmission. So, a switchable tuning resistor $\left(\mathrm{R}_{1}\right.$ in Fig. 1 ) used to reduce the quality factor of the reader coil for wideband communications, and to increase it for power transfer, is introduced. Such switchable capability performance can be seen in the simulation results depicted in Fig.5.

The difficulty on having a well-matched network for both cases, results from the fact that the coupling coefficient from both antennas may vary. This is due not only to the peculiarity that for WPT the distance between both coils needs to be short in order to increase the coupling, but also due to some misalignment.

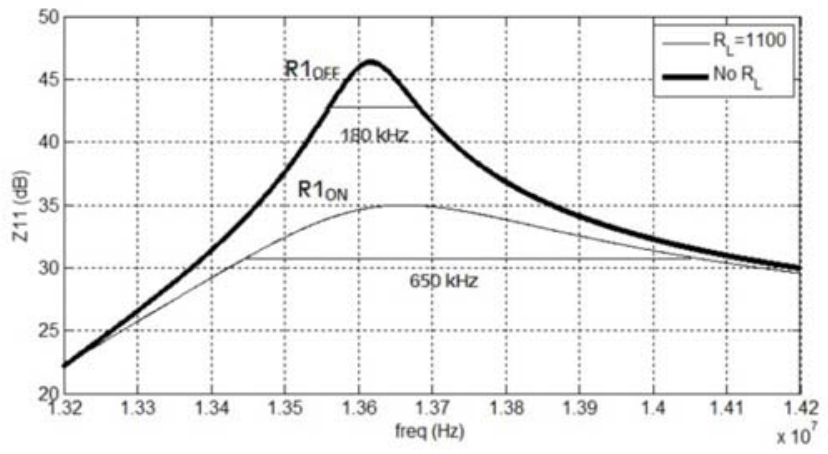

Fig. 5. Input impedance at antenna port, presented when the tunable resistor is switched $\mathrm{ON}$ and $\mathrm{OFF}$

At resonance, the imaginary impedance of the matching network becomes constant, only changing when we move away from resonance. The real part tends to increase by increasing the coupling factor, as will be deduced next.

Using $\mathfrak{R} e\left[Z_{r}^{p r i}\left(\omega_{0}\right)\right]=R_{r}^{p r i}$, as the real part of the impedance seen by the reduced circuit to the primary, the efficiency $\eta$ can be analyzed by using the active components on the equivalent circuit (Fig. 1), and the reflection coefficient $\Gamma$ in our case:

$$
\eta^{p r i}=\frac{R_{r}^{p r i}(k)\left[1-\Gamma^{2}(k)\right]}{R_{r}^{p r i}(k)\left[1-\Gamma^{2}(k)\right]+2 R_{P A}}
$$

In the case of having a not well-matched condition $\left(k \neq k_{o}\right)$, one can define the relation

$$
\Gamma(k)=\frac{R_{r}^{p r i}(k)-R_{r}^{p r i}\left(k_{0}\right)}{R_{r}^{p r i}(k)+R_{r}^{p r i}\left(k_{0}\right)}
$$

which is the reflection coefficient at the primary that will depend on the coupling factor. As can be seen in Fig. 5, by varying $k$, the resonance peeks are affected. The power generated at the receiver (tag or battery), will depend on such matching conditions. In case of WPT, the power provided to the battery will become:

$$
P_{b a t}=\frac{V_{L}^{2}}{4 R_{L}}\left[1-\Gamma^{2}(k)\right]=\frac{V_{L}^{2}}{4 R_{L}}\left[\frac{2 R_{r}^{p r i}\left(k_{0}\right)}{R_{r}^{p r i}(k)+R_{r}^{p r i}\left(k_{0}\right)}\right]
$$

\section{1) NFC transformed impedance}

In NFC communications, the link between transmitter and receiver is normally achieved by means of using parallel tuning capacitors. The NFC receiver equivalent impedance for this system can be obtained from (see secondary circuit in Fig.1):

$$
Z_{N F C}=R_{S}+j \omega L_{S}+\frac{1}{\frac{1}{R_{i c}}+j \omega\left(C_{5}+C_{I C}\right)}
$$

This impedance being transformed into the primary side of the link (transformer) will become $Z_{T}^{p r i}=\frac{(M \omega)^{2}}{Z_{N F C}}$, which at the resonance frequency, and taking the real part, will derive in the following expression: 


$$
\mathfrak{R} e\left\{Z_{N F C}^{p r i}\left(\omega_{0}\right)\right\}=\frac{\left(M \omega_{2}\right)^{2}\left(R_{I C}+R_{1}\right)}{R_{1}^{2}+\left(L_{s} \omega_{0}\right)^{2}}=k^{2} \frac{L_{p}}{L_{s}} R_{I C}
$$

when considering $Q>>1$. It is possible to represent the transformed resistance as a function of the coupling coefficient, expressing $\mathfrak{R} e\left\{Z_{N F C}^{\text {pri }}\left(\omega_{0}\right)\right\}=R_{N F C_{r}}$

$$
R_{N F C_{r}}(k)=R_{1}+k^{2} \frac{L_{p}}{L_{s}} R_{I C}
$$

which can be used in expression (7) to obtain the reflection coefficient for the NFC system. This one is an indicator of the circuit efficiency, for $R_{s}=R_{2}$ :

$$
\Gamma_{N F C}(k)=\frac{k^{2}-k_{0}^{2}}{\frac{2 R_{2}}{R_{I C}}+k^{2}+k_{0}^{2}}
$$

\section{2) WPT transformed impedance}

In the case of using the reader as a WPT, the receiver part of the charging device is normally designed with series LC circuit. In such a case the following expression is deduced for the impedance transformed into the primary side:

$$
\mathfrak{R} e\left\{R_{W P T_{r}}(k)\right\}=\frac{(M \omega)^{2}}{R_{s}+R_{L}} \simeq \frac{k^{2} L_{p} L_{2} \omega_{0}^{2}}{R_{L}}
$$

when $R_{L}>>R_{s}$, where $R_{L}$ is the series resistance seen by the tag or WPT circuit coil. In this case one can deduce the transformed resistance part of the impedance as a function of coupling factor as:

$$
R_{W P T_{r}}(k)=R_{W P T_{r}} \simeq \frac{k^{2} L_{p} L_{2} \omega_{0}^{2}}{R_{L}}
$$

So the reflection coefficient deduced for this scenario will become the following:

$$
\Gamma_{W P T}(k)=\frac{k^{2}-k_{0}^{2}}{\frac{2 R_{L}}{Q_{11} Q_{10} R_{2}}+k^{2}+k_{0}^{2}}
$$

where $Q_{10}=\frac{L_{p} \omega_{0}}{R_{1}}$, is the quality factor without any coupling effect from the secondary coil, and $Q_{11}=\frac{L_{p} \omega_{0}}{R_{1}+R_{T}}$ is the quality factor of the reader with presence of a tag or NFC device $\left(R_{T}\right.$ is the transformed resistance from secondary to primary coil). In all these expressions, there is a dependency on $k$, showing the sensitivity of the efficiency to position. For WPT, the position can be much defined by means of mechanical design, but for NFC the position can vary significantly. In Fig. 6 one can see how the input reflection coefficient at the reader input can vary with $k$ (distance and misalignment).

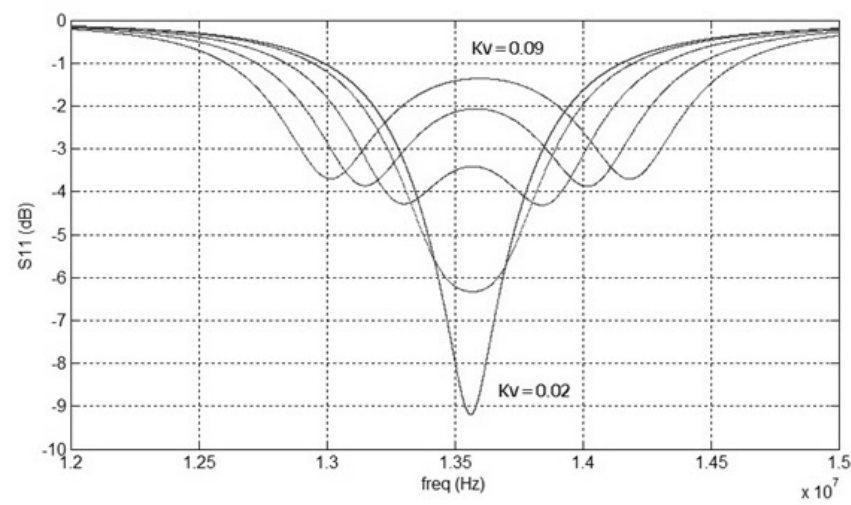

Fig.6 Input reflection coefficient when varying $k$ values 0.02 to 0.09 .

\section{DESIGN IMPLEMENTATION}

Because a WPT system can be seen as being formed from two leakage inductance transformer model tank and series resonant capacitors, it has a current-source characteristic, so it is convenient to connect it to a capacitive filter, having a voltage-source characteristic. This is desirable in some cases as ours, where constant voltage source is required for charging purposes.

To use the secondary leaking inductor as charging coil, the adopted solution is to include a full-bridge rectifier, series connected to the leaking inductor by means of a resonance series capacitor, and with a capacitive output filter that provides a DC voltage at the output load resistance $R_{L}$ (see Fig.7).

According to (10) and (13), there is an opposite dependency of primary impedance versus $R_{L}$ and $L_{s}$. So, in case $R_{L}$ increases, the impedance seen by the reader will decrease for the WPT configuration and will increase for NFC, while in case $L_{s}$ tends to increase, the resistance at the reader will increase for WPT while for NFC it will decrease.

The circuit topology presented to compensate for the tradeoff is depicted in Fig.6. There is a shunt resistor in parallel with the coil $\left(R_{\text {tune }}\right)$ that will be switched ON/OFF accordingly to the functionality. In each case, when the NFC link requires wide bandwidth, the parallel resistor will be connected (switch ON), so the quality factor of the antenna is reduced. For WPT, when transferring power to a remote device, the resistor will be disconnected (switched OFF) increasing automatically the quality factor to its maximum.

The concept presented can be also used when different data rates would be requested. The different $Q$ values would then be achieved by means of adding extra switchable resistances.

To improve the resistance of the circuit versus possible miss-tuning effects (miss-alignment, proximity of elements to the magnetic coupled circuits), tuning capacitors are also included in parallel with the coil inductance, as seen in Fig.7 in the case of our design. By doing so, it is possible to tune to the resonance frequency of the NFC system, helping in maintaining the resonance frequency versus deviation in the antenna environment, and also contributing in improving matching stability (Fig.7). This situation is being justified in 


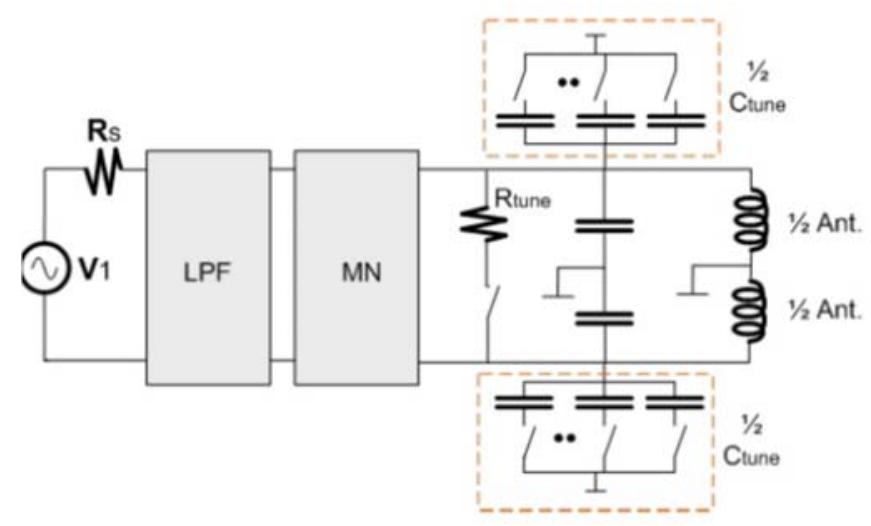

Fig.7 Tuning circuit implementation for antenna resonance and quality factor including LPF and Matching Network (MN). Both signal paths are shown to represent the differential signal path.

NFC, since antenna operates in a difficult environment such as smart-phone, in close proximity with other devices and where some parasitic effects due to unknowns for the placement and locations of components within the mobile phone can be relevant.

\section{MEASUREMENT RESUlTS}

The system under test uses a NFC transmitter with polling tag inventory instruction, to detect from the proximity possible tags. Such scan is streaming continuously the information over a carrier in pulses of $130 \mathrm{~ms}$ and pause intervals of $75 \mathrm{~ms}$. Such system with additional resistance in the tuning circuit $\left(R_{\mathrm{ON}}\right)$ is working perfectly by means of the reader used (based on AMS AS3991 reader IC) since it scans any NFC tag in close vicinity, achieving high rates.

In addition, the same transmitter is used to couple transmitted pulses in the charging receiver. The receiver uses these pulses to charge the battery, extracting the energy from the pulsed HF magnetic field. To do so, the bandwidth is switched to narrow band using the designed tuning circuit, the switching resistor ( $R_{\text {tune }}$ in Fig.8) is commuted by means of a low-voltage single FET bus switch SN74CBTLV1G125 ( $\mathrm{U}_{1}$ in Fig.8). The full bridge high speed rectified is composed by $D_{1}$ to $D_{4}$ diodes (1N4148) and filtered by $C_{l}(33 \mu \mathrm{F})$. Resistors $R_{l}$ and $R_{2}$ are calculated to properly bias the NPN transistors, considering the emitter current of $Q_{1}$ as the charging current for the LiPo batteries. A measurement of such condition can be observed in Fig.9. High current is induced in the secondary when charging configuration is activated, while the current transferred is lower when circuitry is prepared for NFC communications (higher bandwidth requirement).

The battery charger is applied in to a $3.7 \mathrm{~V} \mathrm{Li}-P o$ cell. When the cell voltage is lower than $3.7 \mathrm{~V}$, it will be charged by pulsed constant currents, corresponding to the magnetic induced current by magnetic field in the coil. Once the cell voltage is reached, the cell is being charged at constant voltage until the battery is filled up to full voltage.

Using the resistive impedance from the WPT receiver seeing at the primary (13), the quality factor of a coil and its relationship with the bandwidth, one can obtain the following relation:

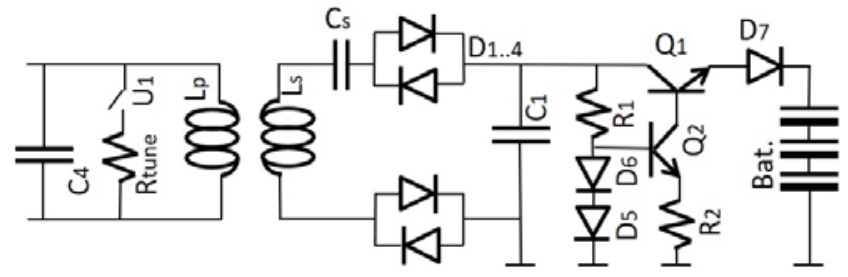

Fig.8 NFC output block diagram, with the wireless energy transfer coils to the pulsed charger circuitry.

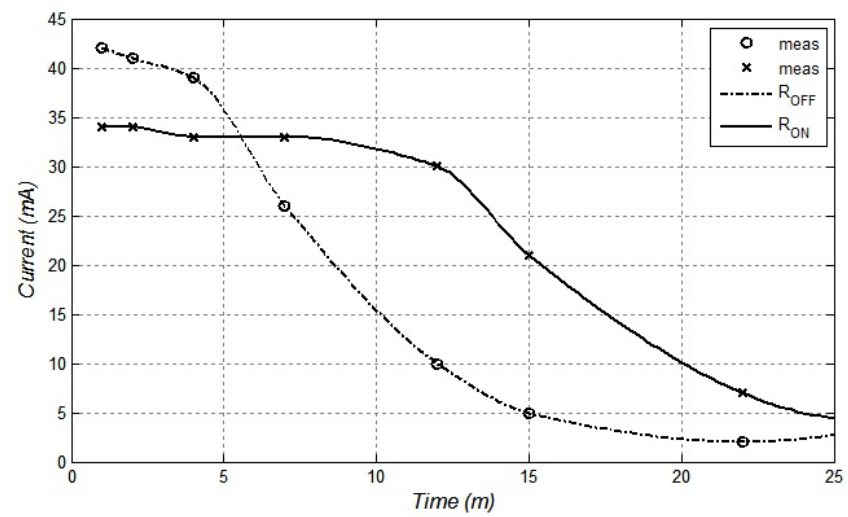

Fig.9 Current charging by the wireless power transfer circuit, with measurement and interpolation curves in the cases of $R_{O N}$ and $R_{O F F}$.

$$
R_{r}^{W}(k)=k^{2}\left(\frac{f_{0}}{B W}\right) \omega_{0}^{2} \frac{R_{p} R_{s}}{R_{L}}
$$

with $R_{p}$ and $R_{s}$ being the series resistance of the coils, Fig.1. In the case of wide bandwidth, the measured delivered pulsed power at the battery is $63 \mathrm{~mW}$. The measurement results extrapolated over an equivalent charging curve for Li-Po battery is traced in Fig.9, where battery is being charged at constant current, provided by transistor $Q_{1}$ (Fig.8), over a period of time. The higher current level corresponds to the situation where switch is OFF and lower current when switch is $\mathrm{ON}$. Voltage curves are extracted from manufacturer.

About the charger circuitry, the voltage to current and control transistors $\left(Q_{1}\right.$ and $Q_{2}$ are BC547 in Fig.8), should be fast enough to react to the NFC pulses defined by the standard protocol. In the case of NFC such speed poses no problem for the pulse intervals, since it obeys to the polling tag inventory duty cycle.

Regarding the reading capability of NFC tags using NFC protocol, the communication is also verified by means of using the commercial NFC IC from AMS (AS3911), with its data framing system for ISO 18092 (NFCIP-1) initiator, ISO 18092 (NFCIP-1) active target, ISO $14443 \mathrm{~A}$ and B reader and FeliCa reader. Such IC includes all the peripheral required to transact communications with any compatible NFC device (mobile phone, passive tag), including the matching network modified with the switching capability. Information is being read from NFC compatible tag card, using NFC mobile phone with an APP that was developed for this testing purposes as well as specific GUI provided by AMS manufacturer. 

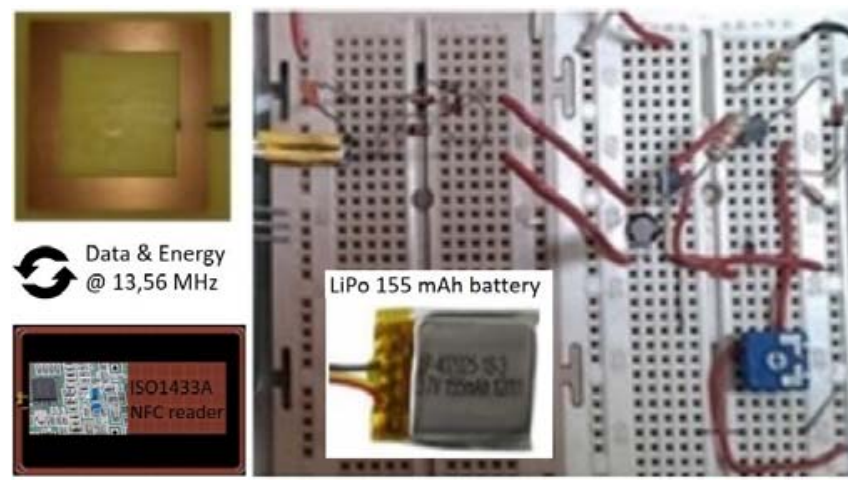

Fig.10 RF to DC charger circuit in discrete components, including information on battery, primary and secondary NFC coils.

\section{CONCLUSION}

Due to the wide spread of mobile phones, there is a trend to increase circuit functionality, which normally goes in the penalty of energy consumption and reduce battery life time. This work presents a solution for using the same circuit as it is used for NFC in a mobile phone, but for the purpose of charging storage elements. The critical elements within the front-end, such as matching network components that affect both the transfer of signal and power from the RFIC driver output (transmitter) to the antenna coils, have been evaluated. We have also presented in detail the trade-off to be able to introduce the proposed solution.

Analyzing the two antenna configurations, one for NFC communications and another for WPT, it has been presented a possible solution to solve the trade-off, when pretending to reuse the same circuitry for both purposes, independently on antenna design and configurations. Finally, a test set up has been implemented to prove the solution including the communications system and Li-Po battery energy storage.

\section{REFERENCES}

[1] A. E. Abdulhadi and R. Abhari, "Multi-Port UHF RFID Tag antenna for enhanced energy harvesting of self-powered wireless sensor", IEEE Transactions on industrial informatics, pp. 1-8, Issue 99, 2015.

[2] A. DeHennis, S.Getzlaff, D.Grice, and M. Mailand, "An NFC-Enabled CMOS IC for a Wireless Fully Implantable GLucose Sensor", IEEE Journal of biomedical and health informatics, Vol. 20, No.1, Jan. 2016.

[3] H- Zhang, J.Li, "NFC in medical applications with Wireless sensors", Electrical and Control Engineering (ICECE); 2011 International Conference on, 16-18 Sept. 2011.

[4] I. Grout, and A.C. Rodrigues, "RFID enabled sensor system design", $5^{\text {th }}$ International Electrical Engineering Congress, Pattaya, Thailand, 8-10 March 2017.

[5] M. Kang, E. Noh, and K. Kim, "NFC transmitter coil placement to minimise degradation of A4WP wireless power transfer efficiency", Electronics Letter, 27th April, Vol.53, No.9, pp.616-618, 2017.

[6] S. Hong, S. Lee, S. Jeon, D.H Kim, J. Song, H. Kim, and J. Kim, "DualDirectional Near Field Communication Tag Antenna with Effective Magnetic Field Isolation from Wireless Power Transfer System", IEEE Wireless Power Transfer Conference, 10-12 May 2017.

[7] G. Vandevoorde and R. Puers, "Wireless energy transfer for standalone systems a comparison between low and high energy applicability", Elsevier, Sensors and Actuators Annals of Physics, vol. 92, pp. 303-311, Aug. 2001.

[8] A. Costanzo, M. Dionigi, F. Mastri, M. Mongiardo, J.A. Russer, and P Russer, "Rigorous design of magnetic-resonant wireless power transfe links realized with two coils", Proceedings of the 44th European Microwave Conference, pp. 414-417, Oct. 2014.
[9] A. Sharma, I.J. Garcia-Zuazola, A. Gupta, A. Perallos, and J.C. Batchelor, "Enhanced H-field in HF RFID systems by optimizing the loop spacing of antennas coils", Microwave and optical technology letters, Vol. 55, pp. 944-948, April 2013.

[10] J.P.K. Sampath, A. Alphones and D.M. Vilathgamuwa, "Tunable metamaterials for optimization of wireless power transfer systems", Antennas and Propagation and USNC/URSI Nation Radio Science Meeting, pp. 107-108, 2015.

[11] C. M. Zierhofer and E. S. Hochmair, "Geometric approach for coupling enhancement of magnetically coupled coils", IEEE Transactions Biomedical Engineering, vol. 43, pp. 708-714, 1996.

[12] D. Rinner, H. Witschnig, and E. Merlin, "Broadband NFC - A system analysis for the uplink", Communication systems, Networks and Digital Signal Processing, 2008 CNSDSP 6th International Symposium, pp.292296, July 2008.

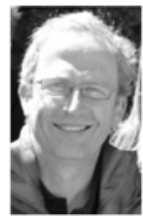

Ignasi Cairó was born in Terrassa (Barcelona), Spain in 1967. He received the electronics and telecommunication degrees by Politechnical Universisty of Catalonia, Barcelona, Spain in 1988 and 1992 respectively.

He joined the research and development department from the group Circutor in 1995, where he was involved in industrial power electronics and RFID systems. From 1999 until 2003 he joined Philips Semiconductors. In Nijmegen, the Netherlands he was developing GSM power amplifiers using BiCMOS process and later in San Jose, California, engaged in the developments of first integrated WLAN transceiver awarded by 2001 price. From 2003 he has been with Epson Electronics $\mathrm{GmbH}$ in Barcelona, leading the $\mathrm{R} \backslash \& \mathrm{D} \mathrm{Lab}$. in RF projects such as antennas, CMOS device characterization, transceiver and system design, from narrow band to ultra-wide band.

At 2010 he joined IREC Institute for Energy Research of Catalonia), as senior researcher and project leader devoted to energy efficiency, microgrids and smart grid technologies, communications and renewable integration activities. Currently involved in wireless communications and RFID systems. He holds 27 patents and a long list of publications in conferences and relevant journals.

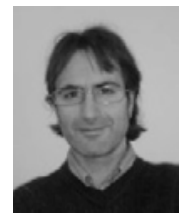

Jordi Bonache was born in Barcelona, Spain, in 1976. He received the physics and electronics engineering degrees and Ph.D. degree in electronics engineering from the Universitat Autònoma de Barcelona (UAB), Barcelona, in 1999, 2001, and 2007, respectively. In 2000, he joined the High Energy Physics Institute,

Barcelona, where he was involved with the design and implementation of the control and monitoring

system of the MAGIC telescope. In 2001, he joined the Department of Electronics Engineering, UAB, where he is currently an Associate Professor. From 2006 to 2009, he was an Executive Manager of CIMITEC, , where he currently leads the research in RFID and antennas. His current research interests include active and passive microwave devices, metamaterials, antennas, and RFID

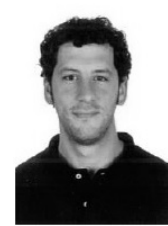

Ferran Paredes, was born in Badalona (Barcelona), Spain in 1983. He received the Telecommunications Engineering Diploma (specializing in Electronics) and the Telecommunications Engineering degree from the Universitat Autònoma de Barcelona in 2004 and 2006, respectively and the $\mathrm{PhD}$ degree in Electronics Engineering from the same university in 2012. He was Assistant Professor from 2006 to 2008 at the Universitat Autònoma de Barcelona, where he is currently working as a Research Assistant. His research interests include metamaterial concepts, passive microwaves devices, antennas and RFID.

Ferran Martín, was born in Barakaldo, Spain, in 1965. He received the B.S. degree in physics and Ph.D. degree from the Universitat Autònoma de 


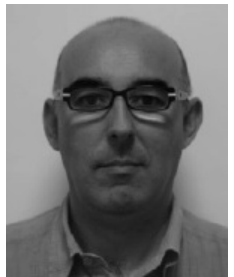

Barcelona (UAB), Barcelona, Spain, in 1988 and 1992, respectively. From 1994 to 2006 he was Associate Professor in Electronics at the Departament d'Enginyeria Electrònica (Universitat Autònoma de Barcelona), and since 2007 he is Full Professor of Electronics. In recent years, he has been involved in different research activities including modelling and simulation of electron devices for high frequency applications, millimeter wave and $\mathrm{THz}$ generation systems, and the application of electromagnetic bandgaps to microwave and millimeter wave circuits. He is now very active in the field of metamaterials and their application to the miniaturization and optimization of microwave circuits and antennas. He is the head of the Microwave Engineering, Metamaterials and Antennas Group (GEMMA Group) at UAB, and director of CIMITEC, a research Center on Metamaterials supported by TECNIO (Generalitat de Catalunya). He has organized several international events related to metamaterials, including Workshops at the IEEE International Microwave Symposium (years 2005 and 2007) and European Microwave Conference (2009), and the Fifth International Congress on Advanced Electromagnetic Materials in Microwaves and Optics (Metamaterials 2011), where he has acted as

chair of the Local Organizing Committee. He has acted as Guest Editor for three Special Issues on Metamaterials in three International Journals. He has authored and co-authored over 500 technical conference, letter, journal papers and book chapters, he is co-author of the book on Metamaterials entitled Metamaterials with Negative Parameters: Theory, Design and Microwave Applications (John Wiley $\backslash \&$ Sons Inc. 2008), author of the book Artificial Transmission Lines for RF and Microwave Applications (John Wiley $\backslash \&$ Sons Inc. 2015), and he has generated $16 \mathrm{PhDs}$. Ferran Martín has filed several patents on metamaterials and has headed several Development Contracts.

Prof. Martín is a member of the IEEE Microwave Theory and Techniques Society (IEEE MTT-S). He is reviewer of the IEEE Transactions on Microwave Theory and Techniques and IEEE Microwave and Wireless Components Letters, among many other journals, and he serves as member of the Editorial Board of IET Microwaves, Antennas and Propagation and International Journal of RF and Microwave Computer-Aided Engineering. He is also a member of the Technical Committees of the European Microwave Conference (EuMC) and International Congress on Advanced Electromagnetic Materials in Microwaves and Optics (Metamaterials). Among his distinctions, Ferran Martín has received the 2006 Duran Farell Prize for Technological Research, he holds the Parc de Recerca UAB - Santander Technology Transfer Chair, and he has been the recipient of two ICREA ACADEMIA Awards (calls 2008 and 2013). He is Fellow of the IEEE since 2012 and Fellow of the IET since 2016 